\title{
Ab-Initio Calculations of Indium Migration in Uniaxial Strained Silicon
}

\author{
Young-Kyu Kim, Soon-Yeol Park, and Taeyoung Won \\ Department of Electrical Engineering, School of Engineering, Inha University \\ National IT Research Center for Computational Electronics \\ 253 YongHyun-dong, Nam-gu, Incheon, Korea 402-751 \\ Tel: +82-32-860-7436, FAX: +82-32-862-1350 \\ kyk@hsel.inha.ac.kr
}

\begin{abstract}
In this paper, we present our ab-initio study on energy configurations, minimum energy path (MEP), and migration energy for neutral indium diffusion in a uniaxial tensile strained $\{100\}$ silicon layer. Our ab-initio calculation of the electronic structure allowed us to figure out transient atomistic configurations during the indium diffusion in strained silicon. We found that the lowest-energy structure $\left(\mathrm{In}_{\mathrm{S}}-\mathrm{Si}_{\mathrm{i}}^{\mathrm{Td}}\right)$ consists of indium sitting on a substitutional site while stabilizing a silicon selfinterstitial in a nearby tetrahedral position. Our ab-initio calculation implied that the next lowest energy structure is $\mathrm{In}_{\mathrm{i}}^{\mathrm{Td}}$, the interstitial indium at the tetrahedral position. We employed the nudged elastic band (NEB) method for estimating the MEP between the two structures. The NEB method allowed us to find that that diffusion pathway of neutral indium is kept unchanged in strained silicon while the migration energy of indium fluctuates in strained silicon.
\end{abstract}

\section{Introduction}

Ion implantation with heavy ion is one of the promising methods for achieving a very shallow junction in microelectronic devices. Due to its heavier mass, indium is considered to be an alternative to boron as a p-dopant in silicon with respect to realizing the shallower and steeper profiles which are required for ultra-large-scale integration (ULSI) technology [1]. Strain effects on dopant diffusion has recently received a lot of attention because strained silicon technology has become very popular for enhancing the mobility of carriers for the next generation metal oxide semiconductor field effect transistor (MOSFET) [2].

As ULSI devices enter the nano-scale era, heavy ion implantation and induced strain methods become more important technologies than former times. On that score, the studies of the properties of indium diffusion in strained silicon would help develop successful process integration. That is because modeling of diffusion profiles of the dopant impurities is imperative for design of the devices due to dopant diffusion in silicon crystal plays a central role. In this work, we investigated the diffusion pathway and energy barrier of indium in an effort to obtain the physical parameters of indium in silicon through $a b$-initio calculations. 


\section{Computational Details}

\subsection{Diffusion Pathway of Indium}

We performed a defect structure calculation in a cubic super-cell, comprising 216 silicon atoms with a single neutral indium atom. The ab-initio calculations were implemented within density functional theory (DFT) with VASP (Vienna Ab-initio Simulation Package) which combines ultrasoft pseudopotentials and generalized gradient approximation (GGA) in the Perdew and Wang formulation. We employed a cutoff energy $E_{\mathrm{c}}=150.62 \mathrm{eV}, 2 \times 2 \times 2$ grid for the $k$-points mesh of Monkhorst-Pack, and a $3 \times 3 \times 3$ simple cubic super-cell (216 atoms). Our optimized silicon lattice constant for GGA in our system is $5.461 \AA$.

We investigated atomic In-Si configuration involved in indium diffusion for searching diffusion pathway of indium. $\mathrm{In}_{\mathrm{s}}-\mathrm{Si}_{\mathrm{i}}^{\mathrm{Td}}$ consists of indium atom sitting on a substitutional site stabilizing a silicon self-interstitial in a nearby tetrahedral position in Fig. 1.

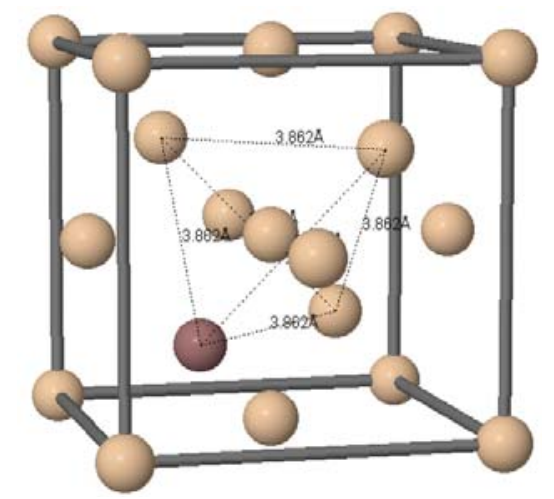

Figure 1: The initial pathway of indium in uniaxial strained $\mathrm{Si}$ is $\mathrm{In}_{\mathrm{s}}-\mathrm{Si}_{\mathrm{i}}{ }^{\mathrm{Td}}$ site : the $\mathrm{In}_{\mathrm{s}}-\mathrm{Si}_{\mathrm{i}}{ }^{\mathrm{Td}}$ configuration, which are the indium atom(red-colored) sits on a substitutional site and stabilizes self-interstitial silicon(yellow-colored) in a nearby tetrahedral position.

$\operatorname{In}_{i}{ }^{\mathrm{Td}}$ is the interstitial indium in the tetrahedral position in Fig. 2. The energy landscape, provided by VASP for In-Si complex indicates that the lowest-energy structure is $\mathrm{In}_{\mathrm{s}}-\mathrm{Si}_{\mathrm{i}}{ }^{\mathrm{Td}}$ and the second lowest-energy structure is $\mathrm{In}_{\mathrm{i}}{ }^{\mathrm{Td}}$. We calculated the energy difference between the first and the second lowest energy configuration is calculated to be $0.492 \mathrm{eV}$. According to the energy landscape, we guess the diffusion pathway of neutral indium is

$$
\mathrm{In}_{\mathrm{S}}-\mathrm{Si}_{i}^{\mathrm{Td}} \rightarrow \mathrm{In}_{i}^{\mathrm{Td}} \rightarrow \mathrm{In}_{\mathrm{S}}-\mathrm{Si}_{i}^{\mathrm{Td}}
$$




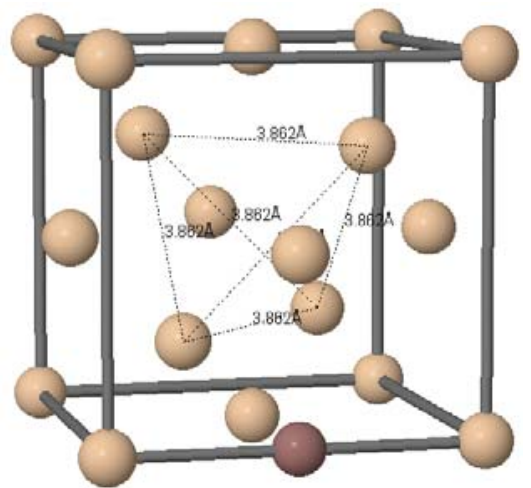

Figure 2: The final pathway of indium in uniaxial strained $\mathrm{Si}$ is $\operatorname{In}_{\mathrm{i}}^{\mathrm{Td}}$ site : the $\operatorname{In}_{\mathrm{i}}^{\mathrm{Td}}$ structure, which is the interstitial indium atom(red-colored) in the tetrahedral position.

\subsection{Minimum Energy Path}

The NEB is an efficient method for finding saddle points and minimum energy paths between the given initial and final states of diffusion [3]. In our previous section, we searched the initial and final states of indium diffusion. The initial state is the lowest energy configuration $\left(\mathrm{In}_{\mathrm{s}}-\mathrm{Si}_{\mathrm{i}}{ }^{\mathrm{Td}}\right)$ and the final state the second lowest energy configuration $\left(\mathrm{In}_{\mathrm{i}}^{\mathrm{Td}}\right)$. Repeating the transitions between the two states, neutral indium diffuses in silicon. Therefore, if we investigate the MEP from the initial state to the final state, we can now obtain the energy barrier for indium migration.

Figure 3 shows the minimum energy path of indium in uniaxial strained silicon calculated by the NEB method with 4 intermediate images wherein stress induced from $0,0.25$ and $0.5 \mathrm{GPa}$, respectively. $\mathrm{Y}$-axis is the migration energy along the MEP of $\mathrm{Si}$ : In from $\mathrm{In}_{\mathrm{s}}-\mathrm{Si}_{\mathrm{i}}{ }^{\mathrm{Td}}$ to $\mathrm{In}_{\mathrm{i}}^{\mathrm{Td}}$.

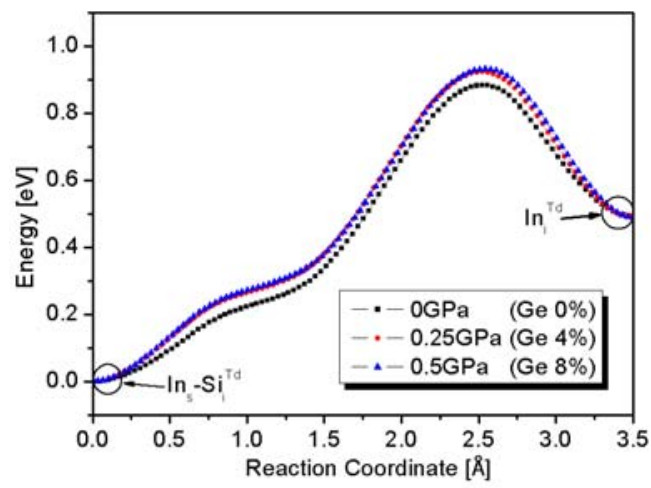

Figure 3: The relative energy along the MEP from $\operatorname{In}_{s}-\mathrm{Si}_{i}{ }^{\mathrm{Td}}$ to $\mathrm{In}_{\mathrm{i}}{ }^{\mathrm{Td}}$ position on the basis of $0,0.25$ and $0.5 \mathrm{GPa}$ stress. 
Figure 4 also shows the MEP of indium in uniaxial strained silicon from inducing 0,1 and $2 \mathrm{GPa}$, respectively. Finally, we arranged migration energy in table 1 with total energies of initial $\left(\mathrm{In}_{\mathrm{s}}-\mathrm{Si}_{\mathrm{i}}{ }^{\mathrm{Td}}\right)$ and final $\left(\mathrm{In}_{\mathrm{i}}{ }^{\mathrm{Td}}\right)$ configurations in 216 atom super cell.

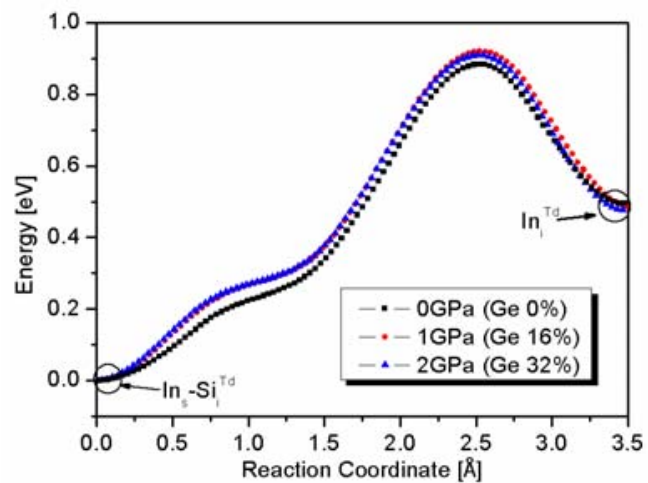

Figure 4: The relative energy along the MEP from $\mathrm{In}_{\mathrm{s}}-\mathrm{Si}_{\mathrm{i}}^{\mathrm{Td}}$ to $\mathrm{In}_{\mathrm{i}}^{\mathrm{Td}}$ position on the basis of 0,1 and $2 \mathrm{GPa}$ stress.

\begin{tabular}{|c|c|c|c|c|}
\hline \multirow{2}{*}{$\begin{array}{c}\text { Stress } \\
(\mathbf{G P a})\end{array}$} & \multirow{2}{*}{$\begin{array}{c}\text { Strain } \\
\text { (ratio by contrast pure Si) }\end{array}$} & \multicolumn{2}{|c|}{ Total energy $(\mathbf{e V})$} & \multirow{2}{*}{$\begin{array}{c}\text { Migration energy } \\
(\mathbf{e V})\end{array}$} \\
\cline { 3 - 4 } & & $\mathrm{In}_{\mathrm{s}}-\mathrm{Si}_{\mathrm{i}}^{\mathrm{Td}}$ & $\mathrm{In}_{\mathrm{i}}^{\mathrm{Td}}$ & \\
\hline 0 & 0 & -1167.6275 & -1167.1357 & 0.884 \\
\hline 0.25 & 0.001603 & -1167.6704 & -1167.1807 & 0.923 \\
\hline 0.5 & 0.003205 & -1167.6778 & -1167.1910 & 0.933 \\
\hline 1 & 0.006410 & -1167.6430 & -1167.1615 & 0.920 \\
\hline 2 & 0.012821 & -1167.4834 & -1167.0088 & 0.910 \\
\hline
\end{tabular}

Table 1: The results of strain, total energy, and migration energy on the basis of stress.

\section{Acknowledgements}

This research was supported by the MIC(Ministry of Information and Communication), Korea, under the ITRC(Information Technology Research Center) support program supervised by the IITA(Institute of Information Technology Advancement) (IITA-2006-C109006030030) and partly by the Korea Institute of Science and Technology Information (KISTI) through the Seventh Strategic Supercomputing Applications Support Program.

\section{References}

[1] P. Alippi, A. L. Magna, S. Scalese, and V. Privitera, Phys. Rev. B 69, 085213, 2004.

[2] B. Sadigh, T. J. Lenosky, M. J. Caturia, A. A. Quong, L. X. Benedict, T. D. Rubia, M. M. Giles, M. Foad, C. D. Spataru, and S. G. Louie, Appl. Phys. Lett. Vol. 80, 4738, 2002.

[3] K. S. Yoon, C. O. Hwang, and T. Won, J. Korean Phys. Soc. 48, 535, 2006. 